\title{
Meson Electroproduction Experiments At Jefferson Lab And The Kaon Form Factor
}

\author{
Tanja Horn ${ }^{* \dagger}$ \\ The Catholic University of America, Washington, DC USA \\ Jefferson Lab, Newport News, VA, USA \\ E-mail: hornt@cua.edu
}

Pion and kaon form factors are of particular interest in our understanding of hadron structure as they are connected to the Goldstone modes of dynamical chiral symmetry breaking. The last decade saw a dramatic improvement in precision of charged pion form factor data and new results have become available on the pion transition form factor. Plans exist to nearly quadruple the momentum transfer over which the charged pion form factor is known. Data on the kaon form factor are sparse, mainly limited to the region that constrains the kaon electromagnetic radius from kaon-atomic electron scattering data. Kaon electro-production cross section data at large virtual photon mass allow one, in principle, to constrain the kaon form factor, although technical difficulties grow as compared to the pion case due to the larger kaon mass and further distance from the pole. Most of the precision cross section measurements at the $6 \mathrm{GeV}$ Jefferson Lab were primarily designed for pions, but some of these experiments also captured kaons in their acceptance. Preliminary kaon cross section results from such experiments show an indication of kaon pole dominance allowing for the first extractions of the kaon form factor from kaon electroproduction data. Planned $12 \mathrm{GeV}$ experiments will further investigate the role of the kaon pole and are anticipated to extend form factor extractions to $Q^{2} \sim 5.5 \mathrm{GeV}^{2}$. The distribution of the fundamental constituents, the quarks and gluons, is expected to be different in pions, kaons, and nucleons. The Electron-Ion Collider with an acceptance optimized for forward physics has the potential for accessing pion and kaon structure functions over a large kinematic region through the Sullivan process. Initial uncertainty projections have been carried out and suggest that such measurements are feasible for both pions and kaons.

The 26th International Nuclear Physics Conference

11-16 September, 2016

Adelaide, Australia

\footnotetext{
* Speaker.

${ }^{\dagger}$ Supported in part by NSF grants 1306227 and 1306418
} 


\section{Introduction - form factors and structure functions}

Pions and kaons occupy a special role in nature [1]. The pion is the lightest quark system, with a single valence quark and a single valence antiquark. It is also the particle responsible for the long range character of the strong interaction that binds the atomic nucleus together. A general belief is that the rules governing the strong interaction are left-right, i.e. chirally, symmetric. If this were true, the pion would have no mass. The chiral symmetry of massless Quantum Chromodynamics (QCD) is broken dynamically by quark-gluon interactions and explicitly by inclusion of light quark masses, giving the pion and kaon mass [1,2]. The pion and kaon are thus seen as the key to confirm the mechanism that dynamically generates nearly all of the mass of hadrons and central to the effort to understand hadron structure [3].

Form factors are the most basic quantity towards understanding light hadron structure. Their importance is evidenced by substantial experimental and theoretical efforts over the last decades [3, $4,5,6,7,8,9,10,11,12,13]$. On the experimental front, dramatic improvement in the understanding of pion electroproduction data $[14,15,17,16,18,19]$ has given confidence in the reliability of the electroproduction method yielding the physical pion form factor. In general, the extraction of precision meson form factors from experiment requires a longitudinal-transverse (L/T) separation of the cross section isolating $\sigma_{L}$, proper selection of the pion pole process, extraction of the form factor using a model, and the validation of the technique.

Recent calculations [3] show that one may expect dominance of hard contributions to the pion form factor for $Q^{2} \sim 8 \mathrm{GeV}^{2}$ [20], where it is predicted that $F_{\pi}$ will start exhibiting precisely the momentum-dependence from QCD. The latter is a power law behaviour plus logarithmic corrections to scaling, but with the normalization fixed by a pion wave function whose dilation with respect to the asymptotic form is a definite signature of Dynamical Chiral Symmetry Breaking (DCSB). Data extending into this regime would thus for the first time have the potential to quantitatively unveil hard QCDs signatures.

The structure functions in terms of sea quarks and gluons are expected to be different for pions, kaons, and nucleons and to provide basic information about their origin of mass. However, experimental data is sparse. It has only been obtained in mesonic Drell-Yan scattering from nucleons in heavy nuclei, with information on the pion's parton distribution functions (PDFs) and results for the ratio of kaon and pion distribution functions. Newer data are needed to address persistent doubts about the large Bjorken- $x$ behaviour of the pion's valence-quark PDF. An approved experiment at $12 \mathrm{GeV}$ Jefferson Lab will use tagged deep inelastic scattering and could contribute to a resolution of the pion question. A similar technique might also serve for the kaon. Looking further ahead, an electron ion collider (EIC) would be capable of providing access to pion and kaon structure functions through measurements of forward nucleon structure functions.

\section{Experimental Determination of Pion and Kaon form factors}

At low values of $Q^{2}, F_{\pi}$ has been determined directly up to photon energies of $Q^{2}=0.253 \mathrm{GeV}^{2}$ at Fermilab and at the CERN SPS from the scattering of high-energy, charged pions by atomic electrons. These data were used to constrain the charge radius of the pion, which is determined to be $r_{\pi}=0.657 \pm 0.012 \mathrm{fm}$. Due to kinematic limitation in the energy of the pion beam and unfavorable 
momentum transfer one has to resort to other experimental methods to reach the higher $Q^{2}$ regime. At higher values of $Q^{2} F_{\pi}$ can be determined from electroproduction of pions on the proton.

In electroproduction experiments one detects the scattered electron and the electroproduced charged pion (or kaon) in coincidence. The differential cross section can be expressed in terms of a known electrodynamic factor and a virtual photoproduction cross section. The latter can be expressed in terms of the linear combinations of the products of virtual-photoproduction helicity amplitudes, which are the unpolarized transverse (T), the purely scalar (longitudinal, L), and the interference terms between the transverse and transverse-scalar states (LT and TT). The dominance of the pion pole in the longitudinal cross section, $\sigma_{L}(\mathrm{~L})$, and its characteristic $t$ dependence allow for extractions of electromagnetic form factors from these data.

Cross section data suggest a dominant pion pole in the longitudinal $\pi^{+}$cross section at values of $-t<0.3(\mathrm{GeV} / \mathrm{c})^{2}$ [21]. The observation of a dominant pion pole alone is, however, not sufficient to make a precise extraction of $F_{\pi}$ from experiment. To minimize background contributions, the longitudinal cross section $\sigma_{L}$, is thus isolated via a Rosenbluth $\mathrm{L} / \mathrm{T}(\mathrm{LT} / \mathrm{TT})$ separation. Without an explicit L/T separation it is not clear what fraction of the cross section is due to longitudinal photons and what the contribution of the pole to it is in these kinematics.

With the availability of high-intensity electron beams, combined with accurate magnetic spectrometers at JLab, it has been possible to determine L/T separated cross sections with high precision. The measurement of these cross sections in the regime of $Q^{2}=0.60-1.60 \mathrm{GeV}^{2}$ (Experiment Fpi-1 [22, 23]) and $Q^{2}=1.60-2.45 \mathrm{GeV}^{2}$ (Experiment Fpi-2 [14] and pionCT [15]) are described in detail in Ref. [24]. Pion electroproduction experiments are performed at the smallest possible value of $t$, which is still a distance away from the pion pole. The pion form factor thus has to be obtained either by kinematic extrapolation or by using a theoretical model. In this method consistency between data and model is essential.

To give confidence in the electroproduction method yielding the physical form factor, one can carry out several experimental studies. These include checking the consistency of the model with the data, extracting the form factor at several values of $t_{\min }$ for fixed $Q^{2}$, verifying that the pole diagram is the dominant contribution to the reaction mechanism, and verifying that the electroproduction technique gives results consistent with those from $\pi-e$ elastic scattering at the same $Q^{2}$. In Ref. [16], to check if the VGL Regge model [25, 26] properly accounts for the pion production mechanism, spectator nucleon, and other off-shell ( $t$ dependent) effects, $F_{\pi}$ was extracted at different distances in $t$ from the pion pole. The resulting $F_{\pi}$ values agree to $4 \%$ and did not depend on the $t$ acceptance, which lends confidence in the applicability of the VGL model to the kinematic regime of the data and the validity of the extracted $F_{\pi}$ values. The dominance of the $t$-channel process in $\sigma_{L}$ was verified in Ref. $[18,19]$ through the charged pion longitudinal cross section ratios, $R_{L}=\sigma_{L}\left[n\left(e, e / \pi^{-}\right) p\right] / \sigma_{L}\left[p\left(e, e / \pi^{+}\right) n\right]$, obtained with a deuterium target. The $t$-channel diagram is purely isovector and so any isoscalar background contributions like $b_{1}(1235)$ to the $t$ channel will dilute the ratio. With increasing $t$, the transverse cross section ratio is expected to approach the ratio of quark charges, i.e., 1/4. The data show that $R_{L}$ approaches the pion charge ratio, consistent with pion-pole dominance. The $t$-dependence of the transverse cross section ratio, $R_{T}=\sigma_{T}\left[n\left(e, e / \pi^{-}\right) p\right] / \sigma_{T}\left[p\left(e, e / \pi^{+}\right) n\right]$, shows a rapid fall-off consistent with $s$-channel quark knockout. Direct comparison of $F_{\pi}$ values extracted from very low $t$ electroproduction with the exact values measured in elastic $\pi-e$ scattering showed that the data are consistent within the 
uncertainties lending further confidence in the validity of the extracted $F_{\pi}$ values.

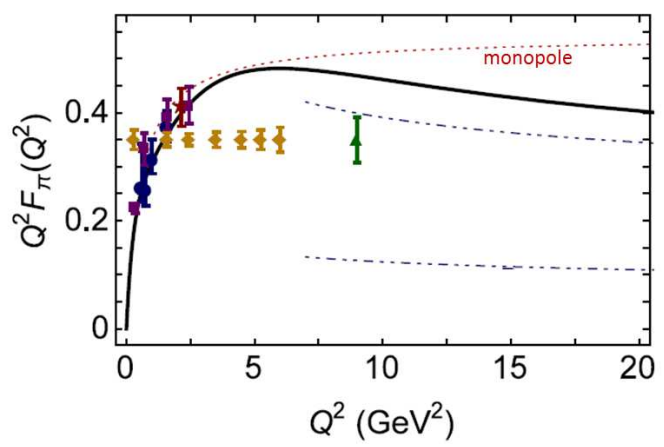

Figure 1: Existing and projected data on the pion form factor. The curves are discussed in the text.

The world precision $F_{\pi}$ data are summarized by the blue and magenta points in Fig. 1. The green point is the projected high- $Q^{2}$ point for $12 \mathrm{GeV} \mathrm{JLab} \mathrm{[27]} \mathrm{taking} \mathrm{into} \mathrm{account} \mathrm{recent} \mathrm{ex-}$ perimental progress. The dotted curve is the maximum-allowed value of the form factor as set by the pion radius. The dot-dot-dashed curves illustrate the calculations from short-distance view assuming either a point-like asymptotic wave function of the pion (lower curve) or a modern wave function (upper curve). The solid curve is the QCD-theory prediction bridging these long- and short-distance domains.

The next simplest meson available for experimental studies and also containing strangeness is the kaon. Of particular interest to hadron structure is the effect of the heavier strange quark in the kaon [28]. Similar to pions the kaon form factor has been determined directly up to photon energies of $Q^{2}=0.10 \mathrm{GeV}^{2}$ at Fermilab [29] and at the CERN SPS [30] from the scattering of highenergy, charged kaons by atomic electrons. These data were used to constrain the mean square charge radius of the kaon, which is determined to be $\left\langle r_{K}^{2}>=0.34 \pm 0.05 \mathrm{fm}^{2}\right.$. In general, the kaon charge radius is expected to be smaller than that of the pion due to the presence of the heavier strange quark. At higher energies the kaon form factor can, in principle, be extracted from kaon electroproduction data. However, there are experimental challenges that have to be addressed. In particular, the kaon pole is farther from the physical region than the pion, which may raise doubts about the ability to obtain reliable information on $F_{K}$ from kaon electroproduction data. Two experimental points are important to consider in this respect. First, extraction of the pion form factor from similar pion electroproduction data at small $t$ is completed by carefully studying the model dependence of the analysis, not by direct extrapolation; and this justifies greater confidence in this method. Second, initial comparative extractions of the pion form factor from low- $t$ and larger- $t$ data suggest only a modest model dependence; and the larger- $t$ pion data lie at a similar distance from the pole as most of the projected kaon data. It is thus reasonable to imagine that $F_{K}$ may be extracted from kaon electroproduction data in a similar fashion, albeit with a larger model dependence than $F_{\pi}$.

Nevertheless, detailed demonstration of the dominance of the kaon pole is required if one is to be confident in the extraction of $F_{K}$. Current electroproduction data show that the $t$-dependence of the longitudinal kaon cross section is less steep than that of the pion $[17,21]$. These data include $\mathrm{L} / \mathrm{T}$ separated cross sections up to photon energies of $Q^{2}=2.35 \mathrm{GeV}^{2}$ from Refs. [31, 32]. The 


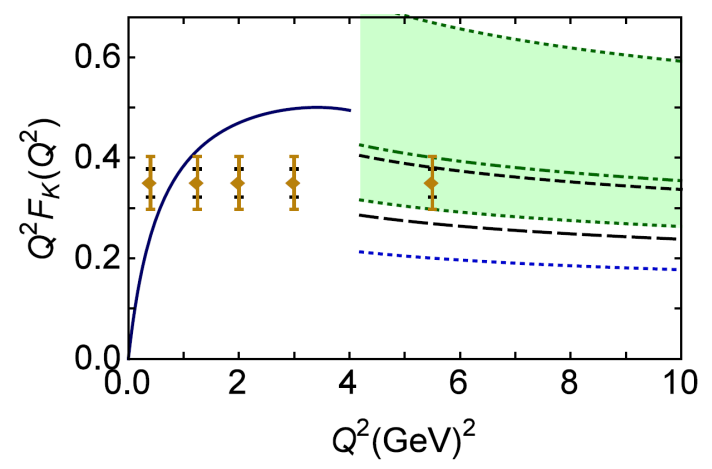

Figure 2: Projected reach for kaon form factor anticipated from a $12 \mathrm{GeV}$ JLab experiment [37]. The error estimates are based on different assumptions about the $t$ - and model-dependence of the form factor extractions, with the larger uncertainty being conservative.The curves are discussed in the text.

results are consistent with the kaon pole factor, $-t /\left(t-m_{K}^{2}\right)^{2}$, giving less enhancement than that of the pion. However, calculations predict a small maximum in the kaon cross section near $t=0.1$ $\mathrm{GeV}^{2}$ due to the kaon pole $[33,34]$. Additional kaon electroproduction data from Jefferson Lab are under analysis [35]. Preliminary results are shown in Fig. 3 and show a trend that may suggest kaon pole behaviour in this kinematic regime.

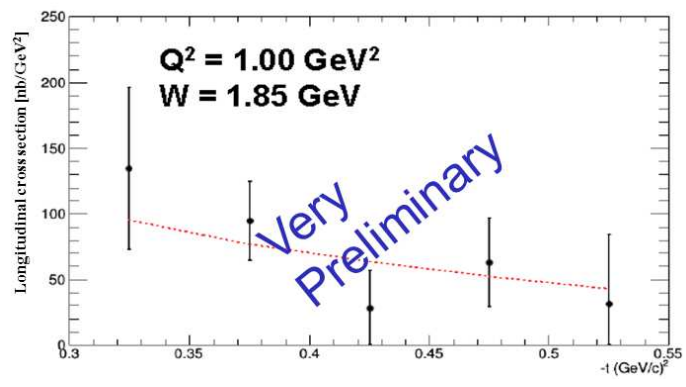

Figure 3: The $t$ depdendence of the longitudinal cross section from an ongoing analysis of kaon electroproduction data from Jefferson $\mathrm{Lab} 6 \mathrm{GeV}$.

Access to small values of $t$ and $\mathrm{L} / \mathrm{T}$ separations of the kaon cross section will be enabled by the $12 \mathrm{GeV}$ Jefferson Lab. The projected reach and accuracy anticipated are shown in Fig. 2. The curves depict theoretical calculations obtained from the hard-scattering formula when different kaon pion distribution amplitudes (PDAs) are used: long-dashed (black) curve, DSE prediction; dot-dashed (green) curve, inferred from lattice QCD values of the lowest two moments, with the green band indicating the uncertainty; dashed (black) curve, obtained from the long-dashed curve by supposing that the second moment of the PDA is just $10 \%$ larger; and dotted (blue) curve, the conformal-limit PDA. The solid (blue) curve is the prediction from Ref. [36]. Further details can be found in Ref. [1]

Current experiments have established the techniques for meson electroproduction experiments and the determination of the meson form factors from these data. The $12 \mathrm{GeV}$ upgrade at the Jefferson Lab features new instrumentation that allows for pushing precision meson form factor measurements to the highest momentum transfers to date. Planned experiments aim for precision 
measurements of the pion form factor to $Q^{2}=6 \mathrm{GeV}^{2}$ and also have the potential to determine the pion form factor up to $Q^{2} \sim 9 \mathrm{GeV}^{2}$ [27]. These measurements are made possible by the combination of the two moderate acceptance, magnetic spectrometers in Hall C. The "High Momentum Spectrometer" (HMS) provides angular acceptance of $6 \mathrm{msr}$ and can detect particles with momenta up to $7 \mathrm{GeV} / \mathrm{c}$. The new "Super-High Momentum Spectrometer" (SHMS) features a solid angle of about $4 \mathrm{msr}$, a momentum coverage up to $11 \mathrm{GeV} / \mathrm{c}$, and covers scattering angles between 5.5 and 40 degrees. The small scattering angle capability combined with excellent control of systematic uncertainties, kinematic reproducibility and well-understood acceptance allows for measurements of precision cross sections and L/T separations at high luminosity $\left(>10^{38} / \mathrm{cm}^{2} \mathrm{~s}\right)$. Such data allow for extractions of the pion form factor at nearly quadruple the momentum transfer over which it is currently known. QCD backgrounds in these data at high values of $Q^{2}$ and $t$ can be addressed experimentally through measurements of the charged pion ratio as discussed above or of the neutral pion cross section. The charged pion $t$-channel diagram is purely isovector. Isaoscalar backgrounds would distort the ratio. The neutral pion production reaction does not have a large pion pole contribution. Measurements of the longitudinal cross section for exclusive neutral pion production like those in Ref. [38] should thus be sensitive to non-pole contributions in the charged pion cross section.

The small-angle capability of the magnetic spectrometers and availability of electron beam energies of $11 \mathrm{GeV}$ will also allow for measurements of the L/T separated kaon cross section at the highest values of $Q^{2}$ to date [37]. The challenge in extracting the kaon form factor from cross section data is that one has to demonstrate that the kaon pole dominates. This can be done in a similar way as for the pion. In addition, the dominance of the kaon pole can, in principle, be tested through the ratio of the longitudinal cross sections of $\sigma^{0}$ to $\Lambda$ channels. The SHMS and HMS missing mass resolution is expected to be very good and together with the spectrometer coincidence acceptance will allow for simultaneous measurements of the $\Lambda$ and $\Sigma^{0}$ channels. If the kaon pole dominates the ratio should be similar to the ratio of the coupling constants $g_{p K \Lambda}^{2} / g_{p K \Sigma}^{2}$.

\section{Insights from Data - Transverse Charge Densities}

The concept of transverse charge densities allows one to relate hadron form factors to their fundamental quark/gluon structure in QCD. They describe the distribution of charge and magnetization in the plane transverse to the direction of motion of a fast hadron. They are related to the partonic picture provided by the Generalized Parton Distributions (GPDs) that encode correlations between longitudinal momentum and transverse position, key properties of the nucleon. GPDs can be understood as spatial densitites at different values of the longitudinal momentum of the quark.

Proton and pion transverse charge densities have been extracted from timelike [39] and spacelike [40, 41, 42] data. In the latter the extension to spacelike domain is accomplished by the use of dispersion relations and models to obtain separate real and imaginary parts. In Ref. [41] the use of models is avoided. This first attempt of a detailed analysis with spacelike data shows that the transverse charge density of the pion is larger than that of the proton close to the core and that the two densities coalesce for impact parameters greater than $0.3 \mathrm{fm}$. The former is expected since the pion radius is smaller than that of the proton. The latter may be interpreted as the proton consisting of a non-chiral core occupying most of the volume and a meson cloud at large impact parameters. 
An ongoing analysis [43] evaluates the world's data on the kaon form factor to extract the corresponding transverse kaon charge density. The available space- and time-like data are examined through a superset with a single global uncertainty, taking into account the individual uncertainties and any differences in the form factor extraction method. The parametrization is based on that used to analyze timelike factor data in Ref. [44], but also includes the newer data from Refs. [45, 46]. The extraction method is based on that used for the proton and pion in Refs. [40] and includes the use of Bessel series expansion and finite radius approximation to determine the impact of experimental uncertainties and the incompleteness error due to the lack of data for $Q^{2}>Q_{\max }^{2}=17.4 \mathrm{GeV}$. Final results will be compared to the pion and proton transverse charge densities allowing for comparison of trends in two and three quark systems.

\section{Pion and kaon structure functions}
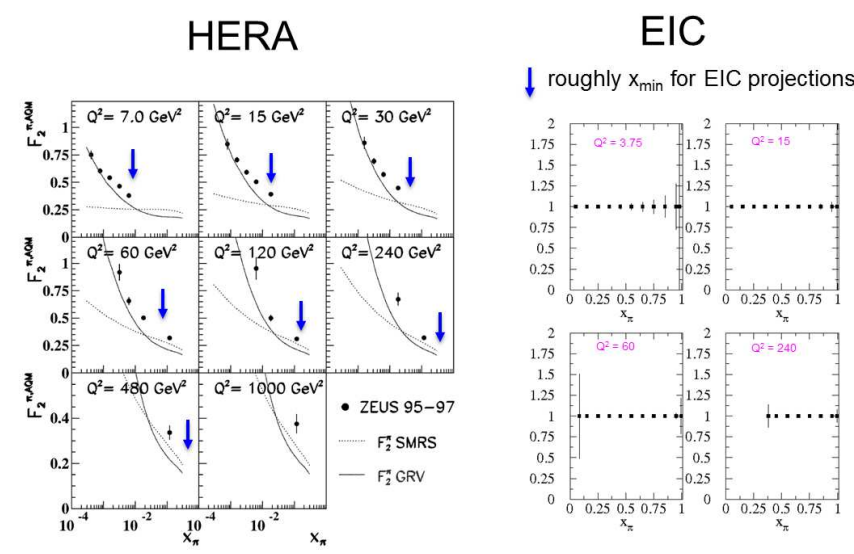

Figure 4: Projected uncertainties of pion structure function measurements at an Electron-Ion Collider using the Sullivan process as a function of $x_{\pi}$ for four values of $Q^{2}$. The electron and ion beam energies were chosen to be $5 \mathrm{GeV}$ and $50 \mathrm{GeV}$, respectively, at a luminosity of $10^{34} \mathrm{~s}^{-1} \mathrm{~cm}^{-2}$. The geometric detection efficiency ( $\sim 20 \%$ for JLEIC) needs to be included for a final uncertainty estimate.

The distribution of the fundamental constituents, the quarks and gluons, is expected to be different in pions, kaons, and nucleons. However, experimental data are sparse. As a result, there has been persistent doubt about the behavior of the pion's valence quark structure function at large Bjorken- $x$ and virtually nothing is known about the contribution of sea quarks and gluons [47]. The Electron-Ion Collider with an acceptance optimized for forward physics has the potential for accessing pion and kaon structure functions over a large kinematic region through the Sullivan process [48]. This would allow for measurements testing if the origin of mass is encoded in the differences of gluons in pions, kaons, and nucleons [2], and measurements that could serve as a test of assumptions used in the extraction of structure functions and the pion and kaon form factors. Measurements at an EIC would also allow to explore the effect of gluons at high $x$. A projection for measurements of the pion structure function through the Sullivan process is shown in Fig. 4. 


\section{Summary}

Meson form factors are the most basic quantity towards our understanding light hadron structure. Precision pion form factor data have become available over the last decade and extensive tests have given confidence in the extraction method. The first extraction of the kaon form factor from electroproduction data has been performed up to $Q^{2} \sim 2 \mathrm{GeV}^{2}$. JLab $12 \mathrm{GeV}$ will dramatically improve the reach of pion (up to $Q^{2}=8.5 \mathrm{GeV}^{2}$ ) and kaon (up to $Q^{2}=5.5 \mathrm{GeV}^{2}$ ) electroproduction data set making possible the extraction of meson form factors and the interpretation of the $12 \mathrm{GeV}$ JLab GPD data. The structure functions in terms of sea quarks and gluons are expected to be different for pions, kaons, and nucleons and to provide basic information about their origin of mass. $12 \mathrm{GeV}$ JLab will provide a first look at the large $x$ region. The planned Electron-Ion Collider provides the opportunity to map pion and kaon structure functions over a wide kinematic range.

\section{References}

[1] T. Horn and C.D. Roberts, J. Phys. 43 (2016) no. 7, 073001.

[2] C.D. Roberts, Perspective on the origin of hadron masses, arXiv:1606.03909 (2016).

[3] L. Chang, I.C. Cloet, J.J. Cobos-Martinez, C.D. Roberts, S.M. Schmidt, P.C. Tandy, Phys. Rev. Lett. 110 (2013) 132001.

[4] W.R. Frazer, Phys. Rev. 115 (1959) 1763.

[5] G.R. Farrar, D.R. Jackson, Phys. Rev. Lett. 43 (1979) 246.

[6] A.V. Efremov, A.V. Radyushkin, Phys. Lett. B94 (1980) 245.

[7] V.A. Nesterenko, A.V. Radyushkin, Phys. Lett. B115 (1982) 410.

[8] S.R. Amendolia et al., Nucl. Phys. B277 (1986) 168; Phys. Lett. B146 (1984) 116.

[9] A.P. Bakulev, A.V. Radyushkin, Phys. Lett. B271 (1991) 223.

[10] R. Jacob, P. Kroll, Phys. Lett. B 315 (1993) 463.

[11] C.D. Roberts, Nucl. Phys. A 605 (1996) 475.

[12] H.B. O’Connell, B.C. Pearce, A.W. Thomas, A.G. Williams, Prog Part. Nucl. Phys 39 (1997) 201.

[13] S. Brodsky, G. deTeramond, Phys. Rev. D 77 (2008) 056007.

[14] T. Horn et al., Phys.Rev.Lett. 97 (2006) 192001.

[15] T. Horn, X. Qian, et al., Phys. Rev. C 78, 058201 (2008).

[16] G. M. Huber, H. P. Blok, T. Horn, et al., Phys. Rev. C 78, 045203 (2008).

[17] T. Horn, Phys. Rev. C 85, 018202 (2012).

[18] G.M. Huber, H.P. Blok, C. Butuceanu, D. Gaskell, T. Horn, D.J. Mack, et al., Phys. Rev. Lett. 112 (2014) $18,182501$.

[19] G.M. Huber, H.P. Blok, C. Butuceanu, D. Gaskell, T. Horn, D.J. Mack, et al., Phys. Rev. C 91 (2015) $18,182501$.

[20] L. Chang, I.C. Cloet, C.D. Roberts, S.M. Schmidt, P.C. Tandy, Phys. Rev. Lett. 111 (2013) 141802. 
[21] L. Favart, T. Horn, M. Guidal, P. Kroll, Eur. J. Phys. A 52 (2016) no.6, 158.

[22] J. Volmer, et al., Phys. Rev. Lett. 86, (2000) 1713

[23] V. Tadevosyan, et al., Phys. Rev. C 75, (2007) 055205

[24] H. P. Blok, T. Horn, et al., Phys. Rev. C 78, 045202 (2008)

[25] M. Guidal, J-M Laget, M. Vanderhaeghen, Nucl. Phys. A 627, (1997) 645

[26] M. Vanderhaeghen, M. Guidal, J-M Laget, Phys. Rev. C 57, (1998) 1454

[27] T. Horn, G. Huber, et al.,Approved JLab experiment E12-07-105: Scaling Study of the L-T Separated Pion Electroproduction Cross Section at $11 \mathrm{GeV}$.

Available online: http://www.jlab.org/exp_prog/proposals/07/PR12-07-103.pdf

[28] P.T.P Hutauruk, I.C. Cloet, A.W. Thomas, Phys. Rev. C 94 (2016) no.3, 035201.

[29] E.B. Dally et al., Phys. Rev. Lett. 45 (1980) 232.

[30] S.R. Amendolia et al., Phys. Lett. B178 (1986) 435.

[31] R.M. Mohring et al., Phys. Rev. C 67 (2003) 055205.

[32] M. Coman et al., Phys. Rev. C 81 (2010) 052201.

[33] S. V. Goloskokov and P. Kroll, Eur. Phys. J. C 65, 137 (2010).

[34] S. V. Goloskokov and P. Kroll, Eur. Phys. J. A 47, 112 (2011).

[35] M.A.P. Carmignotto, $\mathrm{PhD}$ thesis (2017).

[36] P. Maris, P. Tandy, Phys. Rev. C 62 (2000) 055204.

[37] T. Horn, G. Huber, P. Markowitz et al., Approved JLab experiment E12-09-011: Studies of the L-T Separated Kaon Electroproduction Cross Section from 5-11 GeV.

Available online: http://www.jlab.org/exp_prog/proposals/09/PR12-09-011.pdf

[38] C. Munoz-Camacho et al. and the Neutral Particle Spectrometer Collaboration, Approved Jefferson Lab experiment E12-13-010: Exclusive Deeply Virtual Compton and Neutral Pion Cross-Section Measurements in Hall $C$.

Available online: https://www.jlab.org/exp_prog/proposals/13/PR12-13-010.pdf

[39] G. A. Miller, M. Strikman, C. Weiss, Phys. Rev. C 83 (2011) 015203; Phys. Rev. D 83 (2011) 013006.

[40] S. Venkat, J. Arrington, G. Miller, X. Zhan, Phys. Rev. C 83 (2011) 015203.

[41] M.A.P. Carmignotto, T. Horn, G. A. Miller, Phys. Rev. C 90 (2014) 025211.

[42] G. A. Miller, Phys. Rev. C 79 (2009) 055204.

[43] N. Mecholsky et al., Kaon transverse charge density from space- and time-like data, Bulletin of the American Physical Society, APS April Meeting 2017, C12.00003.

[44] C. Pedlar et al., Eur. Phys. J C39 (2005) 41.

[45] T.K. Pedlar et al., Phys. Rev. Lett 95 (2005) 261803.

[46] K.K. Seth et al., Phys. Rev. Lett 110 (2013) 022002.

[47] R.J. Holt, C.D. Roberts, Rev. Mod. Phys. 82, 2991 (2010).

[48] J.D. Sullivan, Phys. Rev. D 5, 1732 (1972). 